\title{
$A b$ initio calculation of structural, electronic and magnetic properties and hyperfine parameters at the Fe sites of pristine $\mathrm{ZnFe}_{2} \mathrm{O}_{4}$
}

\author{
J.J. Melo Quintero ${ }^{a}$, C.E. Rodríguez Torres ${ }^{a}$, L.A. Errico ${ }^{\text {a, b, * }}$ \\ a Departamento de Física, Facultad de Ciencias Exactas, Universidad Nacional de La Plata (UNLP) and Instituto de Física La Plata, IFLP-CONICET CCT-La \\ Plata, 1900, La Plata, Argentina \\ ${ }^{\mathrm{b}}$ Universidad Nacional del Noroeste de la Provincia de Buenos Aires(UNNOBA), Monteagudo 2772, 2700, Pergamino, Buenos Aires, Argentina
}

\section{A R T I C L E I N F O}

\section{Article history:}

Received 18 October 2017

Received in revised form

11 January 2018

Accepted 15 January 2018

\section{Keywords:}

ZFO

Magnetic behavior

Electronic structure

Spin configuration

\begin{abstract}
A B S T R A C T
In this work we present an ab initio study of structural, electronic, magnetic and hyperfine properties of pristine $\mathrm{Zn}$-ferrite $\left(\mathrm{ZnFe}_{2} \mathrm{O}_{4}\right.$, ZFO). Density Functional Theory calculations were performed using the FullPotential Linearized Augmented Plane Waves (FPLAPW) method in the framework of the Generalized Gradient (GGA) and the GGA+U approximations. In order to discuss the magnetic ordering and the electronic structure of the system we considered different spin arrangements. We found that ZFO presents an energy landscape characterized by a large number of metastable states separated by an energy barrier of about $\mathrm{K}_{\mathrm{B}} T_{\mathrm{F}}$, being $\mathrm{K}_{\mathrm{B}}$ the Boltzmann constant and $T_{F}$ the freezing temperature, indicating that ZFO can be described as an spin-glass system at low temperature $(<10.5 \mathrm{~K})$. Our calculations also support the picture that below $10.5 \mathrm{~K}$ small ferromagnetic spin-clusters (short-range interactions) surrounded by similar spin-clusters with opposite spin orientations (long-range interactions) coexist. Finally, our calculations predict a band gap of normal ZFO of $2.2 \mathrm{eV}$ and successfully describe the hyperfine properties (isomer shift, magnetic hyperfine field and electric field gradient tensor) at the Fe sites that are seen by Mössbauer Spectroscopy (MS) at 4.2 and $300 \mathrm{~K}$. This comparison enables us to characterize the local spin structure around $\mathrm{Fe}$ atoms and to explain the origin of the two hyperfine interactions experimentally observed, giving support to the coexistence of short- and a long-range order below $10.5 \mathrm{~K}$.
\end{abstract}

() 2018 Elsevier B.V. All rights reserved.

\section{Introduction}

Oxides with the spinel structure $\left(\mathrm{XY}_{2} \mathrm{O}_{4}\right.$, with $\mathrm{X}$ : $\mathrm{Zn}, \mathrm{Co}, \mathrm{Ni}, \mathrm{Al}$ $\mathrm{Mg}$, Ti, and Y: Al, Ga, In, Ti, V, Cr, Fe, Co, Rh, Mg, Mn, Ni, Cu, etc., see Ref. [1]) comprise an important class of compounds with a variety of electronic and magnetic properties that make them interesting for different technological applications [2-5]. A particular subgroup of this family are the ferrites $\left(\mathrm{XFe}_{2} \mathrm{O}_{4}\right)$, an important and interesting class of magnetic oxides and one of the most extensively studied magnetic materials. Due to their electronic characteristics (small band-gap semiconductors or insulators at low temperatures) they have been used in a number of technologically applications such as microwave devices, magnetic and magneto-optic recording, and electronic information mass storage [5-8].

\footnotetext{
* Corresponding author. Departamento de Física, Facultad de Ciencias Exactas, Universidad Nacional de La Plata (UNLP) and Instituto de Física La Plata, IFLPCONICET CCT-La Plata, 1900, La Plata, Argentina.

E-mail address: errico@fisica.unlp.edu.ar (L.A. Errico).
}

In particular, the $\mathrm{Zn}$-ferrite, $\mathrm{ZnFe}_{2} \mathrm{O}_{4}$ (ZFO) has been the subject of several studies because of its intriguing magnetic properties compared to other spinel ferrites [9]. The ZFO crystal structure is characterized by 8 tetrahedral $A$ sites, occupied by $\mathrm{Zn}^{2+}$ and 16 octahedral $B$ sites occupied by $\mathrm{Fe}^{3+}$ ions (normal spinel) [1]. The magnetic coupling between the Fe atoms on the $B$ sublattice is due to superexchange via oxygen atoms and is weak. The Fe sites form a corner-sharing tetrahedral lattice that has the same atomic arrangement of pyrochlores and C15 Laves phase. These systems are characterized by geometrical frustration and unusual ground state phases (spin glass, spin ice, etc. [10]). Considering the similarity of the structures, topological frustration is expected in normal ZFO [11,12].

Different groups have studied the magnetic properties of ZFO using various experimental methods until now, but contradicting experimental results have been reported. Usually it is claimed that ZFO ferrite is a strongly paramagnetic substance with antiferromagnetic coupling only below $T_{N}=10.5 \mathrm{~K}$ (Neel temperature). But the situation is more complex. Neutron diffraction studies performed on high quality samples [13] have shown that even at 
temperatures of about $100 \mathrm{~K}$, a short-range order (SRO) develops. Below $10.5 \mathrm{~K}$ long-range antiferromagnetic order (LRO) and SRO coexist and the system remains magnetically disordered even at temperatures as low as $1.5 \mathrm{~K}$ [13]. In consequence, the well-defined cusp-like anomaly at around $10.5 \mathrm{~K}$ in static susceptibility data does not correspond to the Neel Temperature in the ordinary meaning. Usa et al. [11,14] suggested that the LRO is driven by the quenched atomic disorder, allowing the interpretation of ZFO as an intrinsically frustrated spin system with nearest-neighbor antiferromagnetic interactions (Ref. [11] and references therein). On the other hand, from static magnetic susceptibility experiments, a positive Curie-Weiss $\left(\theta_{C W}\right)$ temperature of $120 \mathrm{~K}$ was estimated [11]. This result is in clear contradiction with the previously mentioned antiferromagnetic interaction between nearest-neighbors in normal ZFO, suggesting that the SRO is ferromagnetic like. This conclusion was supported by a theoretical analysis (based on a classical Heisenberg spin model) of neutron scattering experiments performed in single-crystal samples $[10,11]$. This work assumes a normal freeof-defects sample and predicts that ZFO is governed by an antiferromagnetic third-neighbor exchange interaction $J_{3}$ and a ferromagnetic first-neighbor one $J_{1}$ [11]. Based in these results, a magnetic configuration with ferromagnetically-coupled spins forming clusters and arranged antiferromagnetically with other clusters could be possible below the cusp temperature. Based on this idea Yamada et al. [11] and Tomiyazu et al. [15] proposed a molecular spin model that is consistent with the neutron scattering data [10]. A spin-molecule refers to a self-organized spin cluster. Correlation among the spins that form the spin-molecule is negligible in comparison to the intra-molecular correlation. The spins of one Fe-tetrahedron of the pyroclore-type ZFO structure are coupled ferromagnetically but antiferromagnetically with the spins of others Fe-tetrahedron [11,15].

Later, M. A. Hakim et al. [16] reported that field cooled and zero field cooled DC magnetizations display a divergence at low temperature. This result indicates that there is a frozen state with freezing temperature $T_{F}=21 \mathrm{~K}$, suggesting that ZFO presents a spin-glass phase at low temperatures and it is not a canted antiferromagnet. A spin-glass is a magnetically disordered system with high magnetic frustration in which each spin freezes in a random orientation below $T_{F}$ [17]. A spin-glass contains many metastable states separated by an energy barrier of about $\mathrm{K}_{B} T_{F}$ [18].

In order to elucidate the magnetic ground state and the electronic structure of pristine normal ZFO, we have performed an $a b$ initio study of the structural, electronic and magnetic properties of this oxide. The calculations used the Full-Potential Linearized Augmented Plane Waves method (FPLAPW). The Generalized Gradient (GGA) and the GGA $+U$ approximations were used to describe the exchange and correlation potential. We discuss the validity of both approaches and the dependence of the results with the value of $U$. To find the lowest energy spin configuration, we considered the ferromagnetic and different antiferromagnetic spin configurations. For all cases, we computed the total energies, the band structures and hyperfine parameters (isomer shift, quadrupole splitting, quadrupole shift and hyperfine magnetic field) at the Fe sites. From the lowest total energy obtained the equilibrium structure and the ground state of pristine ZFO is found. The hyperfine parameters at the Fe sites for the different magnetic configurations studied were compared with the experimental ones, obtained in Mössbauer Spectroscopy (MS) experiments performed on high quality samples of normal ZFO. As we will show, the two different interactions experimentally detected at $5 \mathrm{~K}$ can be associated to Fe nucleus in two different magnetic environments, one ferromagnetic and the other antiferromagnetic, in agreement with the spin-glass phase and the formation of small ferromagnetic spinclusters surrounded by clusters with opposite spin orientation.

\section{The system under study}

The spinel ferrites $\left(\mathrm{XFe}_{2} \mathrm{O}_{4}\right)$ are isostructural with the mineral $\mathrm{MgAl}_{2} \mathrm{O}_{4}$ (spinel) [1] and belong to the space group $\mathrm{Fd} 3 m$ (Oh7). Ferrites crystallize in a face-centered cubic lattice with a closepacked arrangement of oxygen ions, with $\mathrm{X}$ and Fe ions at two different crystallographic sites (sites $A$ and $B$ ). These $A$ and $B$ sites have $\mathrm{O}_{4}$ (tetrahedral) and $\mathrm{O}_{6}$ (octahedral) oxygen coordination, respectively [1]. The unit cell contains eight formula units. Two types of ferrites can be distinguished: the normal and inverted ferrites. In the normal ferrites, the $\mathrm{X}$ ions occupy the $A$ sites and the $\mathrm{Fe}$ atoms the $B$ sites. In the inverted ferrites, eight Fe atoms are located at the $A$ sites and the $\mathrm{X}$ and Fe metal ions occupy the $B$ sites in equal proportions. Inverted ferrites can be described by the formula $\mathrm{Fe}[\mathrm{FeX}] \mathrm{O}_{4}$.

The spinel structure is characterized by two parameters, the lattice constant $a$ and the oxygen positional parameter $u$. For an ideal spinel $u$ has the value of 0.25 for the $-3 m$ origin [1]. Generally, $u$ is often found to be higher than the above value for all the spinels. The spinel ferrite investigated here, $\mathrm{ZnFe}_{2} \mathrm{O}_{4}$ (ZFO), adopts the normal spinel structure. However, depending on the procedure under which ZFO is growth, partial inversion can occur. For ZFO, different values of the lattice parameter $a$ have been reported, ranging from 8.43 to $8.46 \AA[13,19-22]$. A lattice parameter $a=8.52$ Å at ambient temperature was determined using x-ray powder diffraction by K. Kamazawa et al. [10]. The authors claim that this value (slightly larger than those previously reported) is more accurate since it was obtained from a high-quality sample. In normal ZFO the $\mathrm{Zn}$ atoms are located at tetrahedral $A$-sites (Wyckoff position $8 a(1 / 8,1 / 8,1 / 8)$ ), whereas the Fe atoms (which carry a magnetic moment due to the partially filled $3 d$ shell) occupy the octahedral $B$-sites only $(16 d,(1 / 2,1 / 2,1 / 2))$. The Fe-O and Zn-O bond lengths are 2.04 and $2.00 \AA$, respectively. The $\mathrm{O}$ atoms are at $32 e(u, u, u)$ positions of the fcc structure $(u=0.258$, Ref. [22]).

\section{Computational aspects}

\subsection{Computational methods}

$A b$ initio electronic structure calculations were employed to determine the self-consistent potential and the charge density inside the cell of ZFO. From the charge density structural, electronic, magnetic and hyperfine properties of ZFO were obtained. The calculations were performed with the WIEN2K [23] implementation of the FPLAPW method [24-26] in a scalar relativistic version. Exchange and correlation effects were treated within densityfunctional theory using the $\mathrm{Wu}$ and Cohen parameterization of the generalized gradient approximation (GGA) [27]. Since the exchange and correlation effects included in GGA are insufficient to describe 3- $d$ transition oxides, GGA plus the Hubbard $U$ term $(\mathrm{GGA}+U)$ in the self-interaction correction (SIC) scheme was employed [28]. GGA $+U$ is not a fully ab initio method, because we have to select $a$ priori the on-site Coulomb energy $U$ [29]. In this study we took $U=5 \mathrm{eV}$ for the $3 d$-Fe orbitals. This value was selected after the study of a set of Fe oxides $\left(\mathrm{FeTiO}_{3}, \mathrm{TiFe}_{2} \mathrm{O}_{4}, \mathrm{FeO}, \mathrm{Fe}_{2} \mathrm{O}_{3}, \mathrm{Fe}_{3} \mathrm{O}_{4}\right)$ as a function of $U$ in the range from plain GGA calculations $(U=0)$ to $U=7.0 \mathrm{eV}$. For $U=5 \mathrm{eV}$, we correctly reproduce the structural properties, band gaps, magnetic moments and experimentally reported hyperfine parameters at the Fe sites for the set of Fe-oxides studied (see next section). Based on this, we "transferred" the value of $U$ to ZFO. For some selected systems, we also studied the effect of adding a $U$ value for the $3 d$ levels of $\mathrm{Zn}$. As we will show, none of the results presented here are affected by this additional correction.

In the FPLAPW method, the unit cell is divided into nonoverlapping spheres with radius $R_{i}$ and the interstitial region. The 
atomic spheres' radii used for $\mathrm{Zn}$ and Fe were $1.06 \AA$ and for the oxygen atoms the radii were $0.8 \AA$. The parameter $R K_{\max }$ controls the size of the basis set and was set to 7. Here $R$ is the smallest muffin-tin radius and $K_{\max }$ the largest wave number of the basis set. We introduced local orbitals to better describe $\mathrm{O}-2 \mathrm{~s}, \mathrm{Fe}-3 p$ and $\mathrm{Zn}$ $3 p$ orbitals [23]. Once self-consistency of the potential was achieved, quantum-mechanically derived forces were obtained, the ions were displaced according to a Newton damped scheme, and the new positions for the atoms were obtained (for details see Ref. [30]). The procedure was repeated until the forces on the ions were below a tolerance value of $0.1 \mathrm{eV} / \AA \AA$. This energy-minimization procedure was performed for all the spin arrangements considered in the present study. Integration in reciprocal space was performed using the tetrahedron method, taking an adequate number of $k$ points (50 points) in the first Brillouin zone.

To check the precision of our calculations, we performed several additional calculations, following the procedure described by some of us in Ref. [31]. In order to have an idea of the error introduced by the choice of the exchange-correlation functional, we performed calculations using the local density approximation (LDA, Ref. [32]) plus $U$ approximation for the exchange and correlation potential (considering $U=5 \mathrm{eV}$ ). Both approximations predicted structural, electronic and hyperfine properties that differ in less than $2 \%$. Once a choice is made for the exchange-correlation functional, the KohnSham equations are fully determined and the code solves it numerically, introducing numerical errors. The parameters that are most relevant for keeping the numerical errors small are the basis set size and the density of the mesh used for Brillouin zone sampling. By examining the effect of different basis sets (we varied $R K_{\max }$ from 6 to 8 ) and $K$-point samplings we conclude that for $R K_{\max }=7$ and $50 \mathrm{~K}$-points numerical errors are negligible and our results are converged in $1 \%$ or less. Another source of uncertainty on the electric field gradient (EFG) is the choice of the lattice parameter. To study the influence of the lattice parameter in our predictions we calculated the different structural, electronic, magnetic and hyperfine properties for several $a$ values in an interval of $\pm 0.8 \%$ around $8.46 \AA$. This interval covers all the experimental $a$ values reported in the literature for ZFO. We found that the variations in the mentioned range of lattice parameters produce changes in the order of $1 \%$ (or less) in band-gaps, magnetic moments and energy differences. In the case of the hyperfine parameters, isomer shifts and hyperfine fields are unaltered by a change of $a$ in the mentioned range. Quadrupole splitings and quadrupolar shifts changes in about $\pm 5 \%$ going from $8.46 \AA$ to $8.52 \AA$ or $8.41 \AA$. Based in all these studies we can infer that our results are very well converged, giving confidence to our conclusions. In particular, energies differences are converged in about $1 \mathrm{meV}$ per unit formula.

\subsection{Hyperfine parameters calculation}

Nuclear techniques, such as Mössbauer Spectroscopy (MS), have been extensively applied in order to determine the subnanoscopic scale environments of impurities or constituent atoms of the system studied (see Ref. [33] and references there in). What is most rewarding in these methods is their "ability" to measure charge symmetry related properties, such as the EFG tensor, as well as magnetic properties, such as magnetic hyperfine fields $\left(B_{H F}\right)$, making it possible to obtain a fingerprint of the electronic and magnetic configuration near and at the probe nucleus [34,35].

At temperatures above $10.5 \mathrm{~K}$ ZFO presents a paramagnetic behavior, Two magnitudes can be determined by MS: the isomer shift (IS), which provides information on the local chemical bond, and the quadrupole splitting $(Q S)$, which is a "fingerprint" of the local symmetry around the probe nucleus. Both quantities depend on the solid state environment. The IS is given by Refs. [34,37]:

$\mathrm{IS}=\alpha\left(\rho_{A}-\rho_{R}\right)$,

where $\rho_{A}$ and $\rho_{S}$ stand for the electron charge densities at the nuclear positions (the contact densities) in two different solid state environments, here denoted the absorber $(A)$ and the reference $(R)$ materials (BCC-Fe), respectively. $\alpha$ is a calibration constant. Its value varied in the range $0.25-0.32$ a.u. ${ }^{3} \mathrm{~mm} \mathrm{~s}^{-1}[38,39]$ for the $14 \mathrm{keV}$ excited state of ${ }^{57} \mathrm{Fe}$. In the present study (GGA+U calculations, $U=5 \mathrm{eV}$ ) we obtained $\alpha$ from a linear regression analysis of calculated electron charge densities (obtained using the same approach for the exchange and correlation potential and the same numerical and computational parameters) as a function of observed isomer shifts for a series of Fe-compounds. The obtained results are shown in Fig. 1. The agreement between experiment and our theoretical predictions may be judged from Fig. 1. In order to perform the linear fit we have used the formalism described by Borcherds, Ngwengwe, and Sheth $[40,41]$. From this linear fit we obtained $\alpha=-0.271$ a.u. ${ }^{3} \mathrm{~mm} \mathrm{~s}^{-1}$ (regression coefficient $R=0.998$ ).

The $Q S$ is originated by the interaction of the nuclear quadrupole moment with the crystalline electrostatic potential. For the case of the $I=3 / 2,14.4 \mathrm{KeV}$ excited state of ${ }^{57} \mathrm{Fe}$, the $Q S$ is given by Refs. [34,37]:

$Q S=\frac{e Q}{2} V_{Z Z}\left(1+\frac{\eta^{2}}{3}\right)^{1 / 2}$

being

$\eta=\frac{V_{X X}-V_{Y Y}}{V_{Z Z}}$

the asymmetry parameter. In the above equations $e$ is the elementary charge and $Q$ is the nuclear quadrupole moment of the $14 \mathrm{keV}$ excited state of the ${ }^{57} \mathrm{Fe}$ Mössbauer probe nucleus [42]. $V_{X X}$, $V_{Y Y}$ and $V_{Z Z}$ are the principal components of the diagonal EFG tensor in a coordinate system with the convention $\left|V_{X X}\right| \leq\left|V_{Y Y}\right| \leq\left|V_{Z Z}\right|$. The EFG tensor is a rank two traceless symmetric tensor whose principal components Vii are defined by the second derivative (with respect to the spatial coordinates) of the Coulomb potential $V(\boldsymbol{r})$

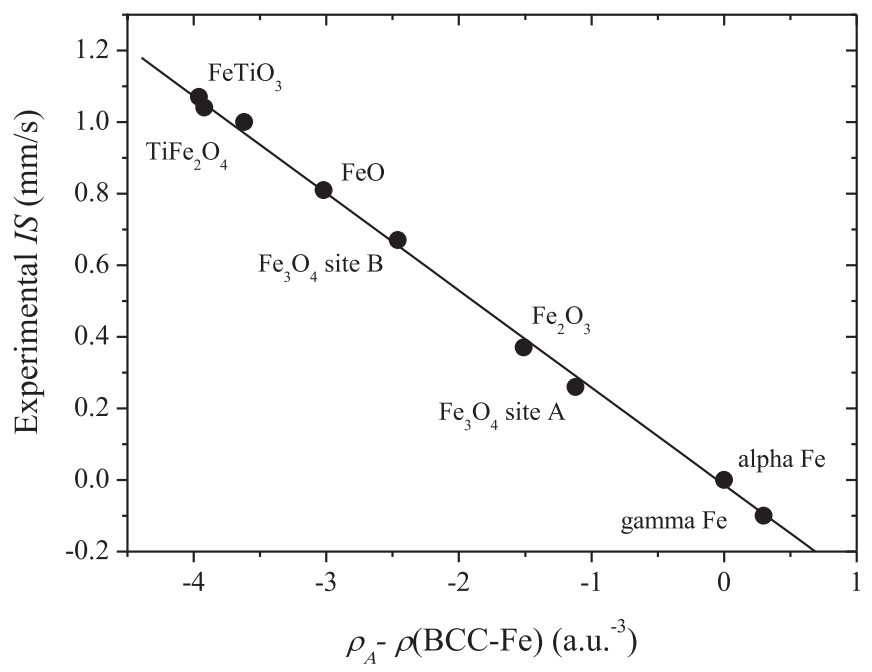

Fig. 1. Experimental isomer shift IS (relative to BCC-Fe) as a function of the difference in electron density $\left(\rho_{\mathrm{A}}-\rho(\mathrm{BCC}-\mathrm{Fe})\right)$ for the different Fe-compounds studied. Solid line corresponds to the linear fit to the data points. All calculations correspond to $U=5 \mathrm{eV}$. 
created by the charge density surrounding a given nucleus. The principal components $V_{i i}$ can be determined straightforwardly once the total charge distribution has been accurately calculated.

For $T<10.5 \mathrm{~K}$, a combined electric and magnetic interaction will be observed and, in addition to the $I S$, the experimentally determined magnitudes are the magnetic hyperfine field $\left(B_{H F}\right)$ and the quadrupole shift $(\varepsilon)$. For the case of $\eta=0$ (as in the present study), $\varepsilon$ at the Fe sites is given by Ref. [37]:

$\varepsilon=\frac{e Q}{8} V_{Z Z}\left(3 \cos ^{2}(\theta)-1\right)$

being $\theta$ the angle between the magnetic hyperfine field $B_{H F}$ and the main component $V_{z z}$ of the electric field gradient tensor. $B_{H F}$ is a fingerprint of the magnetic configuration and the spin polarization near and at the probe nucleus. $B_{H F}$ can be expressed as a vectorial sum of three components: the dipole term, the orbital term and the Fermi contact term $\left(B_{C}\right)$. For iron compounds, the orbital and dipolar terms are much smaller than the Fermi contact one (the contact term, originates from the nonzero electron spin density at the ${ }^{57} \mathrm{Fe}$ nuclei, accounts for more than $90 \%$ of $B_{H F}$ ). So, in good approximation we can write $B_{H F}=B_{C}$.

It is known that the use of DFT with local or semi-local approximations for the exchange and correlation potential proved to be very valuable for the understanding of different properties of solids. But this scheme presents some several shortcomings when applied to magnetic systems. One of them is the (several) underestimation of $B_{C}$ at the site of $3 d$ metal $[43,44]$. The reason of this failure is related to insufficient account of core level spinpolarization. This problem is of course observed for Fe. In the past several attempts were done to correctly calculate $B_{C}$ at the Fe nuclei [43-46]. Compared to $B_{C}$, the magnetic moments of Fe atoms are in much better agreement with the experiments. This circumstance was used by Novak and Chlan [47] to obtain in a semiempirical way a correction to $B_{C}$. In this model, the spin magnetic moments of the Fe-3d $\left(\mu_{3 d}\right)$ and the valence Fe- $4 s\left(\mu_{4 s}\right)$ electrons are obtained and $B_{C}$ is expressed as their linear combination.

$B_{C}=A \mu_{3 d}+B \mu_{4 s}$

The coefficients $A$ and $B$ of the linear combination were calculated by comparison with the hyperfine field experimentally determined in a number of iron compounds. From this study, Novak and Chlan obtained $A=-16.92 \mathrm{~T} / \mu_{B}$ and $B=1229 \mathrm{~T} / \mu_{B}$ [47]. In the present work we will use eq. (5) in conjunction with the previously indicated values of $A$ and $B$ to obtain $B_{H F}$. We have to mention that we prove the validity of this approach performing GGA $+U$ calculations $(U=5 \mathrm{eV})$ of $B_{H F}$ at Fe sites in different Fe oxides.

To show the effect of including $U$ for the Fe- $3 d$ states, we present in Table 1 the hyperfine properties at the Fe sites calculated using plain GGA and GGA $+U$ for $\mathrm{Fe}_{2} \mathrm{O}_{3}, \mathrm{Fe}_{3} \mathrm{O}_{4}$, and $\mathrm{TiFe}_{2} \mathrm{O}_{4}$. As can be seen, the $\mathrm{GGA}+U$ calculations predict hyperfine parameters in better agreement with the experiments [48] than those obtained in the framework of GGA. Also, GGA $+U$ predicts band gaps that are in the order of $2.0-2.2 \mathrm{eV}$ and magnetic moments in the order of $\pm 4.00-4.20 \mu_{B}$, in good agreement with the experiments. GGA predicts band gaps in the order of $0.2 \mathrm{eV}$ or metallic character in some Fe-oxides and magnetic moments in the order of $\pm 3.5-3.7 \mu_{B}$.

\section{Results and discussion}

\subsection{Magnetic structure and lattice parameter}

As mentioned before, pristine ZFO was generally considered an antiferromagnetic insulator below $10.5 \mathrm{~K}$, but further experimental
Table 1

$A b$ initio predictions for the hyperfine parameters at the Fe sites in some Fe oxides obtained using GGA and GGA $+U(U=5 \mathrm{eV})$. Experimental results can be found in Ref. 48. In the cases of $\mathrm{Fe}_{3} \mathrm{O}_{4}$ and $\mathrm{TiFe}_{2} \mathrm{O}_{4}$ the two lines refers to the interaction associates to Fe in sites $A$ (first lines) and $B$ (second lines).

\begin{tabular}{llllll}
\hline System & & $B_{H F}(\mathrm{~T})$ & $I S(\mathrm{~mm} / \mathrm{s})$ & $\varepsilon(\mathrm{mm} / \mathrm{s})$ & $\Delta Q(\mathrm{~mm} / \mathrm{s})$ \\
\hline $\mathrm{Fe}_{2} \mathrm{O}_{3}$ & $\mathrm{GGA}$ & 46.8 & 0.57 & & 0.43 \\
& $\mathrm{GGA}+U$ & 53.5 & 0.44 & & 0.30 \\
& Exp. $(4.2 \mathrm{~K})$ & 54.2 & 0.49 & & 0.23 \\
$\mathrm{Fe}_{3} \mathrm{O}_{4}$ & $\mathrm{GGA}$ & 46.2 & 0.36 & 0.0 & \\
& & 46.8 & 0.73 & 0.06 & \\
& $\mathrm{GGA}+U$ & 53.1 & 0.29 & 0.0 & \\
& & 50.8 & 0.64 & 0.1 & \\
& Exp. (300 K) & 49 & 0.26 & 0.0 & \\
$\mathrm{TiFe}_{2} \mathrm{O}_{4}$ & $\mathrm{GGA}$ & 46 & 0.67 & 0.02 & 0.2 \\
& & 45 & 0.77 & & 0.9 \\
& $\mathrm{GGA}+U$ & 41 & 0.91 & & 3.1 \\
& & 41 & 0.96 & & 2.6 \\
& Exp. (4.2 K) & $20 \pm 4$ & 1.05 & & 2.5 \\
& & $35 \pm 5$ & 1.2 & & \\
\hline
\end{tabular}

results revealed that the behavior of ZFO below $10.5 \mathrm{~K}$ is complex, and its ground state is still an open question [11-15]. In order to study this question we have considered the ferromagnetic case and different antiferromagnetic spin arrangements in the ZFO unit cell. In all cases a cubic supercell cell with 56 atoms was constructed from the 14-atoms primitive cell. The first spin arrangement (AF1) is shown in Fig. 2a and 2b. As can be seen, the AF1 structure is formed by spin chains with two orientations crossing each other along the lattice. The second structure (AF2, Fig. 2c and d) consists of a pair of spins aligned ferromagnetically but antiferromagnetically to another pair from the nearest layer. The other 3 arrangements (AF3, AF4, AF5, Fig. 2e-j) consist of random distributions of 8 Fe atoms with majority spin and $8 \mathrm{Fe}$ atoms with minority spin in the $16 \mathrm{~B}$-sites of the structure (we anticipate here that ferrimagnetic solutions have larger energies that those with a null net magnetic moment). For the study of systems that require the description of the structure of crystals with magnetic ordering, in which the positions of the atoms and also the orientation of the magnetic moments of each atom need to be specified, the importance of the symmetry becomes clear. In our case, it is important to note that the symmetry of the magnetic structures AF2, AF3, AF4, and AF5 is smaller than the crystal space group, showing the complexity of the ZFO system. In Table 2 we present the spin orientation of each Fe atoms in all the spin configurations reported in this work.

From the total energy obtained for each magnetic configuration as a function of the lattice constant $a$ (see Fig. 3) we found that the lowest energy states correspond to antiferromagnetic cases and, of all of the antiferromagnetic configurations studied here, the lowest energy corresponds to the AF2 spin arrangement (see Fig. 2). This result supports the picture presented in Refs. [11,15] for the magnetic structure of ZFO, of ferromagnetic spin-clusters surrounded by similar clusters with opposite sign. The study of magnetic configurations characterized by clusters of more than $2 \mathrm{Fe}$ atoms with the same spin in the $x, y$, and $z$ directions (with the condition of a resulting antiferromagnetic system) would be of great interest but, unfortunately, for the study of these spin-clusters, supercells with a very large number of atoms are necessary. The combination of large number of atoms in the supercell and the precision required, make this study unaffordable. Preliminary results obtained considering a 112-atoms supercell confirm that clusters of more than two Fe atoms have slightly lower energies (or, at least, the same energy within convergence errors) than AF2.

For the AF2 arrangement our $\mathrm{GGA}+U(U=5 \mathrm{eV})$ calculations 
a)
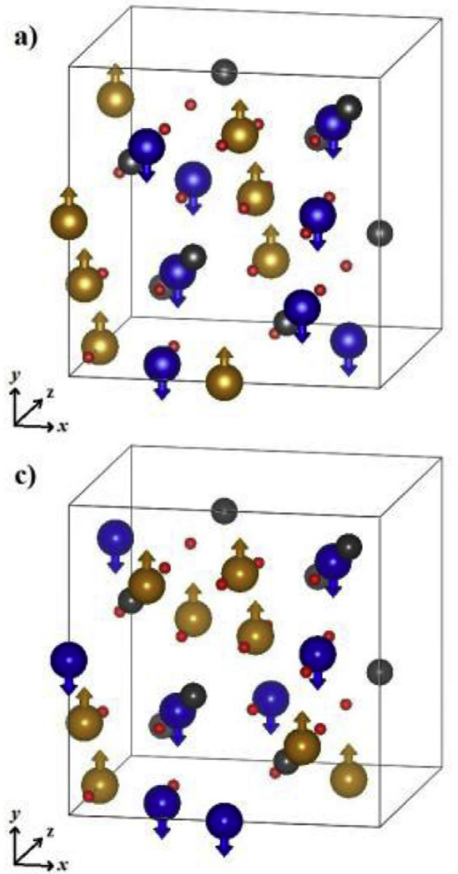

e)

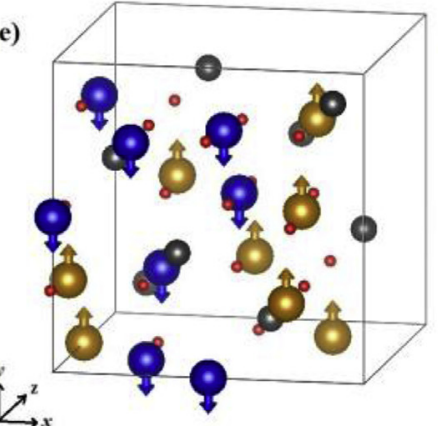

g)
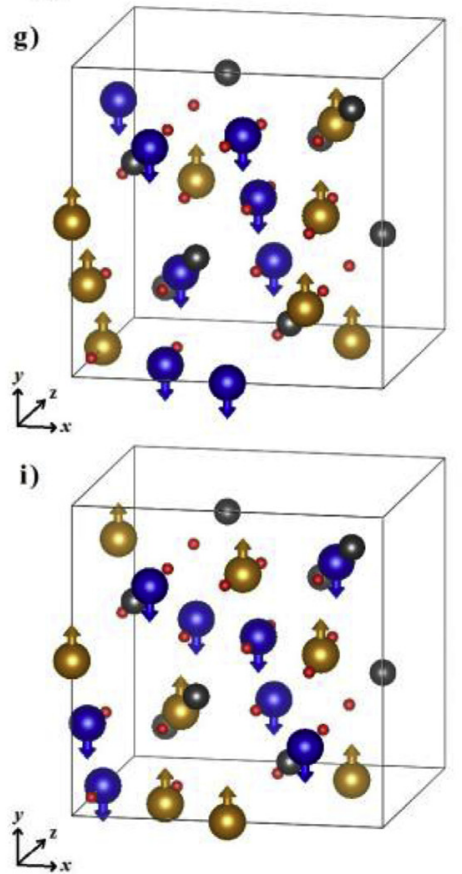

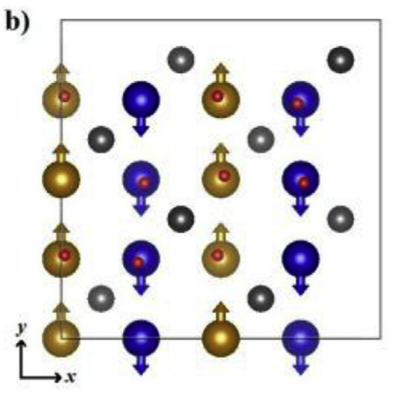

d)

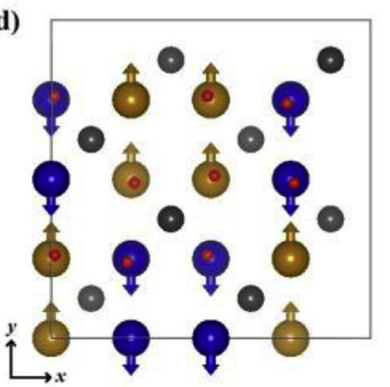

f)

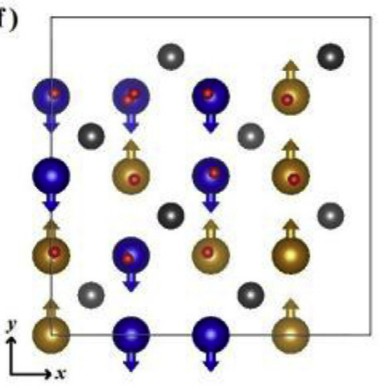

h)

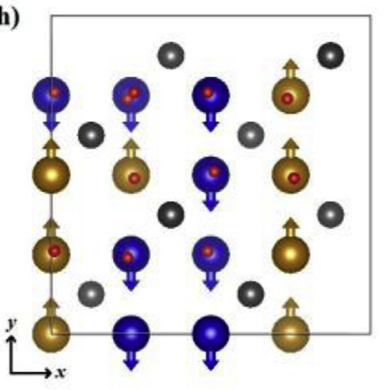

Fig. 2. The different antiferromagnetic spin arrangements studied here. Figure a) and b) show the AF1 arrangement in two different perspectives. Figures c) and d), e) and f), g) and h), i) and j) shows the AF2, AF3, AF4 and AF5 arrangements in two perspectives,

Table 2

Spin configuration for all the arrangements studied here. $+(-)$ indicates majoritary (minoritary) spin channels.

\begin{tabular}{lllllll}
\hline Fe-atom at: & Ferro & AF1 & AF2 & AF3 & AF4 & AF5 \\
\hline $0.5 ; 0.5 ; 0.5$ & + & + & + & - & - & + \\
$0.5 ; 0.25 ; 0.25$ & + & + & + & + & - & + \\
$0.0 ; 0.0 ; 0.5$ & + & + & + & + & + & + \\
$0.0 ; 0.75 ; 0.25$ & + & + & + & - & - & - \\
$0.0 ; 0.5 ; 0.0$ & + & + & - & - & + & - \\
$0.0 ; 0.25 ; 0.75$ & + & + & - & + & + & + \\
$0.5 ; 0.0 ; 0.0$ & + & + & - & - & - & - \\
$0.5 ; 0.75 ; 0.75$ & + & + & - & - & - & - \\
$0.75 ; 0.75 ; 0.5$ & + & - & - & + & + & + \\
$0.25 ; 0.5 ; 0.25$ & + & - & - & + & + & + \\
$0.25 ; 0.25 ; 0.5$ & + & - & - & - & - & - \\
$0.75 ; 0.0 ; 0.25$ & + & - & - & + & + & - \\
$0.25 ; 0.75 ; 0.0$ & + & - & + & - & - & + \\
$0.75 ; 0.5 ; 0.75$ & + & - & + & + & + & - \\
$0.75 ; 0.25 ; 0.0$ & + & - & + & + & + & + \\
$0.25 ; 0.0 ; 0.75$ & + & - & + & - & - & - \\
\hline
\end{tabular}

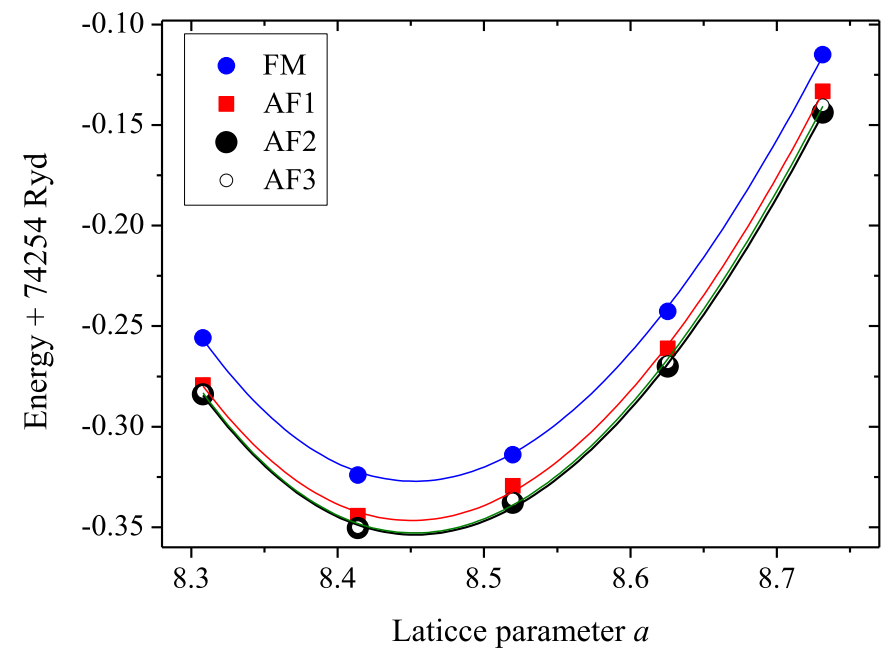

Fig. 3. Calculated energy as a function of the lattice parameter $a$ (in $\AA$ ) of ZFO for different spin configurations. The minimum energy lattice parameter corresponds to $8.46 \AA$, independently of the considered spin arrangement. The lowest energy is obtained for the AF2 configuration. For simplicity, AF4 and AF5 were not included in the figure (the energy differences between configurations AF3, AF4, and AF5 are included in the size of the points).

predict $a=8.458 \AA, u=0.2600$ and magnetic moments at the Fe sites $\mu(\mathrm{Fe})$ of $\pm 4.19 \mu_{B}$ (no spin polarization was found at the $\mathrm{Zn}$ sites, while the magnetic moment at the oxygen atoms is smaller than $\pm 0.05 \mu_{B}$ ). These theoretical results are nearly independent of the antiferromagnetic spin arrangement considered (see Table 3) and are in very good agreement with the experimental ones (a lattice parameter value between 8.43 and $8.46 \AA$ [19-21], $u=0.258$ [22], $\mu(\mathrm{Fe})=4.2 \mu_{B}$ [13]). Plain GGA calculations predict $a=8.38 \AA$, $u=0.261$ and $\mu(\mathrm{Fe})= \pm 3.6 \mu_{B}$. When $U=5 \mathrm{eV}$ is applied to $\mathrm{Zn}-3 d$ states, none of the discussed properties are changed: $a=8.457 \AA$, $u=0.2604$ and magnetic moments at the Fe sites $\mu(\mathrm{Fe})$ of $\pm 4.20 \mu_{B}$ (again, no spin polarization was found at the $\mathrm{Zn}$ sites). In conclusion, the $U$ correction for the Fe-3d states is fundamental for a correct prediction of the band gap, the equilibrium volume and the

respectively. Golden and blue spheres represent the two orientations of the Fe atoms in each configuration. The small red spheres oxygen atoms and the grey spheres the $\mathrm{Zn}$ ones. Arrows also indicates the spin orientations up or down). (For interpretation of the references to colour in this figure legend, the reader is referred to the Web version of this article.) 
Table 3

Energy (in units of eV per unit formula, u.f.) and magnetic moment at the Fe sites $\mu(\mathrm{Fe})$ for the different magnetic arrangement studied for the case of pristine normal ZFO. Energies are referred to the ferromagnetic case. A more negative value for $\Delta E$ indicates a more stable arrangement. A third decimal was included in the case of $a$ in order to see how the predictions differs for each magnetic arrangement.

\begin{tabular}{llll}
\hline Magnetic arrangement & $\Delta E(\mathrm{meV} /$ u.f. $)$ & $a(\AA)$ & $\mu(\mathrm{Fe})\left(\mu_{B}\right)$ \\
\hline FM & 0.00 & 8.453 & +4.22 \\
AF1 & -29 & 8.457 & \pm 4.20 \\
AF4 & -41 & 8.456 & \pm 4.20 \\
AF3 & -42 & 8.456 & \pm 4.20 \\
AF5 & -44 & 8.457 & \pm 4.19 \\
AF2 & -46 & 8.458 & \pm 4.19 \\
\hline
\end{tabular}

magnetic moment at the Fe sites. On the other hand, the application of a second corrective $U$ value to the $\mathrm{Zn}-3 d$ states not produces any noticeable change in the structural properties of ZFO and the magnetic moment at the Fe sites (changes are smaller than our convergence error).

In Table 3 we present the results obtained for the energies of each spin-arrangement, referred to the ferromagnetic case in units of eV/unit formula (u.f.). For a simple comparison, the energies are referred to the ferromagnetic case. As can be seen, the energy difference between the five antiferromagnetic configurations is very small and comparable to the barrier potential given by $K_{B} T_{F}$, being $T_{F}$ the spin freezing temperature $(21 \mathrm{~K}$, see Ref. [16]). This point reinforces the experimental observations that showed that normal ZFO is a three-dimensional spin frustrated system and there is no long-range magnetic ordering in ZFO at temperatures as low as $1.5 \mathrm{~K}[13]$ and presents an spin-glass behavior [10,12,15].

To conclude, we studied the effect of flipping only one spin in the AF2 magnetic structure. We calculated the total energy of this system in order to check the energy difference between this configuration and AF2. We obtained that the magnetic moment of the "flipped" Fe changes from -4.2 to $+4.2 \mu_{B}$ and, in consequence, the resulting cell has a total magnetic moment of $8.4 \mu_{B}$. The energy difference between AF2 and this system $\left(\Delta E=E^{A F 2}-E^{f l i p}\right)$ is -10 $\mathrm{meV} /$ u.f. This result ensures that pristine ZFO has an antiferromagnetic configuration. Larger negative differences were obtained when more than one Fe atoms were flipped. Similar results were obtained for the other magnetic arrangements.

\subsection{Electronic structure. Density of states (DOS)}

The density of states (DOS) of pristine ZFO obtained in the GGA calculations are shown in Fig. 4 for the different configurations studied here Because the configurations AF2, AF3, AF4, and AF5 present very similar DOS we show here only the result obtained for AF2. The DOS and the overall band structure obtained are consistent with previous theoretical results obtained using GGA [49,50]. As can be seen in Fig. 4, the GGA calculations predict that ferromagnetic ZFO has a metallic character. The configuration AF1 is still metallic, but a band gap is opened for the lowest energy configurations (AF2, AF3, AF4, and AF5). For the case of AF2 (the lowest energy configuration), this band gap is in the order of $0.4 \mathrm{eV}$. These results show that the symmetry break due to spin alignment plays a role in the band structure of ZFO as was also reported by Soliman et al. [50]. In that work the authors studied the band structure of ZFO decreasing the octahedral point group symmetry of Fe atoms. For decreasing symmetries a semiconducting trend is observed for antiferromagnetic structures. On the other hand, Singh et al. [49] found that ferromagnetic and antiferromagnetic structures were metallic, but they only studied the highly symmetric AF1 arrangement in the framework of the GGA approximation.

A significant opening of the gap (compared to the GGA value of

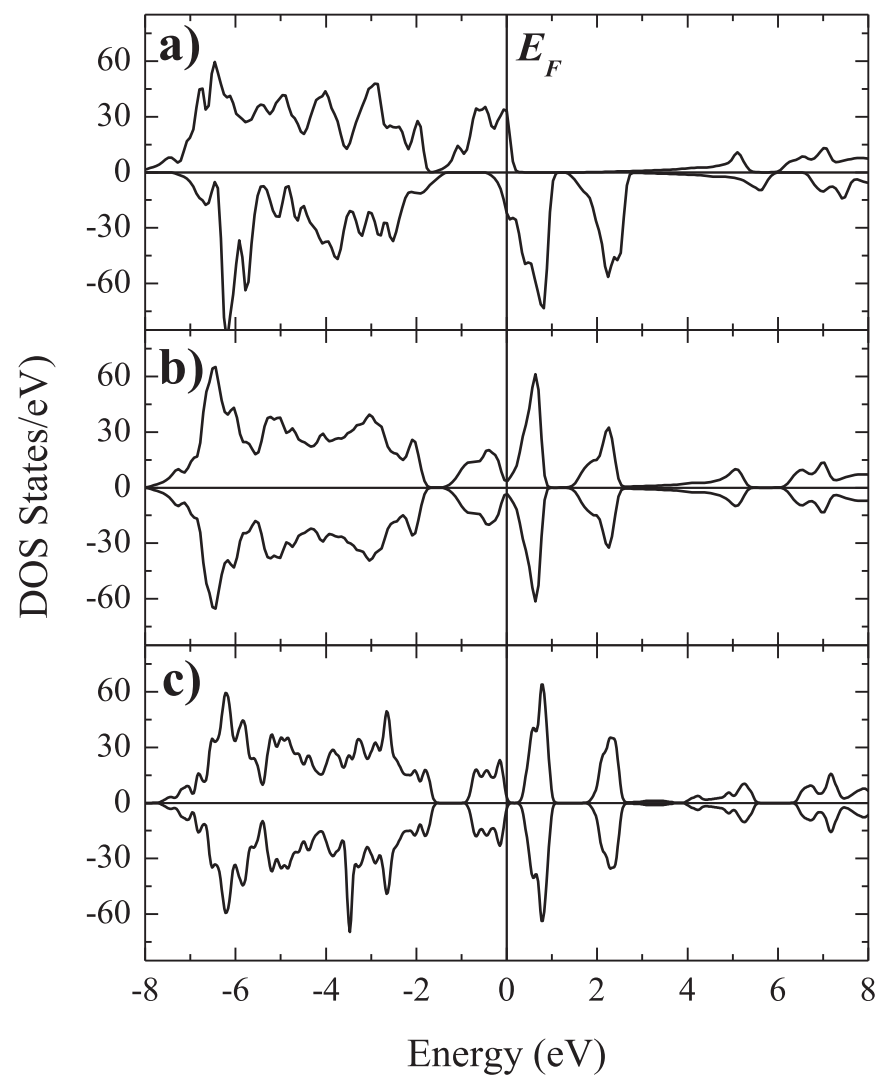

Fig. 4. Total density of states (DOS) of ZFO obtained in the GGA calculations for the ferromagnetic (a), AF1 (b) and AF2 (c) spin arrangements. Energies are referred to the Fermi level $\left(E_{F}\right)$, denoted as a vertical line.

$0.4 \mathrm{eV}$ ) is found using GGA $+U$. Varying $U$ (applied to Fe-3d states) from 0 to $6 \mathrm{eV}$, the band gap increases linearly with $U$. For the case of the lowest energy structure (AF2), a band gap of $2.2 \mathrm{eV}$ is obtained for $U=5 \mathrm{eV}$ (see Fig. 5). This result is opposite to that reported by Soliman et al. [50], who stated that for $U$ in the range $1-6 \mathrm{eV}$ there is not a significant energy band gap and a half metallic character is found for $U$ values as large as $6.0 \mathrm{eV}$. Smaller band gaps were obtained in our calculations for the ferromagnetic and the AF1 configurations.

Experimental results report that ZFO is an insulator with a band gap in the order of $2.0 \mathrm{eV}$ [51], but band-gaps as low as $0.2 \mathrm{eV}$ have been inferred from its temperature dependence in the paramagnetic state $[1,52,53]$. The band gap obtained in the plain GGA calculation is twice as large than the experimental value of $0.2 \mathrm{eV}$. Taking into account that DFT typically underestimates the band gap in oxide systems, we claim that the reported value of $2.0 \mathrm{eV}$ for the band gap is the most reliable value. In order to avoid the use of empirical factors and to unravel the controversies, we also performed calculations using the Tran-Blaha modified Becke-Johnson (TB-mBJ) [54,55] approximation for the exchange and correlation potential to explore the band gap correction. This exchange and correlation potential yields band gaps with an accuracy comparable to approaches which are orders of magnitude more expensive in time and computational resources. The TB-mBJ leads to a band gap of $2.3 \mathrm{eV}$ (see Fig. 6). This result confirms our result obtained using a $U$ value of $5.0 \mathrm{eV}$ and is in agreement with the experimental works that reported band gaps in the order of $2.0 \mathrm{eV}$.

In Fig. 5, we also present the partial DOS (PDOS) of each of the constituent atoms obtained in the GGA $+U(U=5 \mathrm{eV})$ calculations. As can be seen, the occupied Fe-3d levels form a band below the 


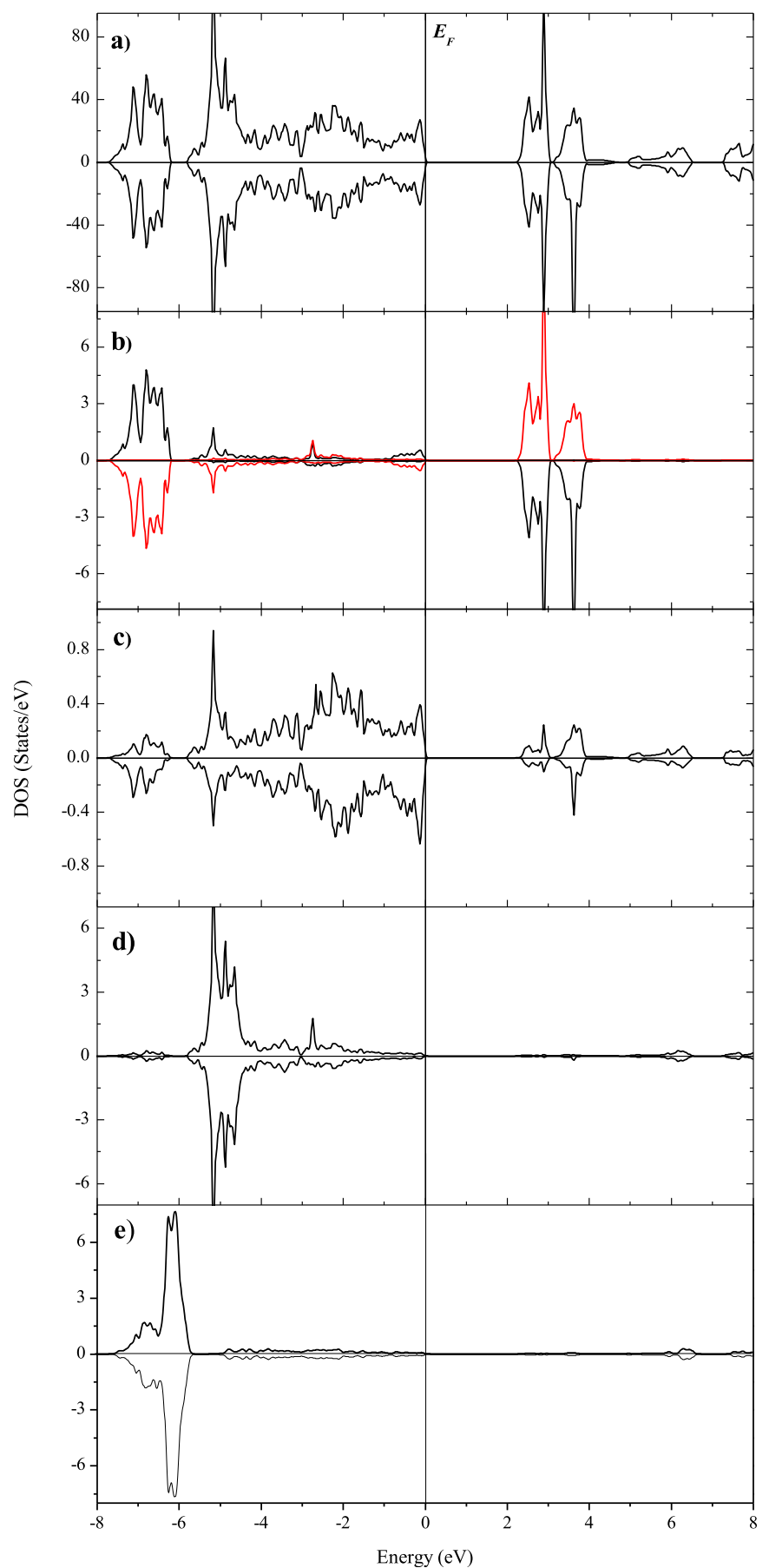

Fig. 5. Total (a) and partial DOS of the dominant angular character of the constituent atoms of ZFO obtained in the GGA $+U(U=5 \mathrm{eV})$ calculations and considering the AF2 spin arrangement (lowest energy case). (b) Fe-3d contribution. One majority (red line) and one minority (black line) Fe-atoms are shown; (c) $0-2 p$ contribution; (d) Zn-3d contribution; e) $\mathrm{Zn}-3 d$ contribution when a $U=5 \mathrm{eV}$ correction is included to describe these states of $\mathrm{Zn}$. Energies are referred to the Fermi level $\left(E_{F}\right)$, denoted as a vertical line. (For interpretation of the references to colour in this figure legend, the reader is referred to the Web version of this article.)

valence band (in the range $-7.6--6.2 \mathrm{eV}$ ). The valence band of ZFO $(-5.8-0 \mathrm{eV})$ is dominated by the $0-2 p$ states; however, there are contributions of $\mathrm{Zn}-3 d$ and Fe-3d states. These hybridization contributions evidence the covalent nature of ZFO. Above the Fermi level, the conduction band has predominantly Fe-3d character with small hybridization of $0-2 p$ states. Similar results were obtained for the case of the TB-mBJ calculations (see Fig. 6).

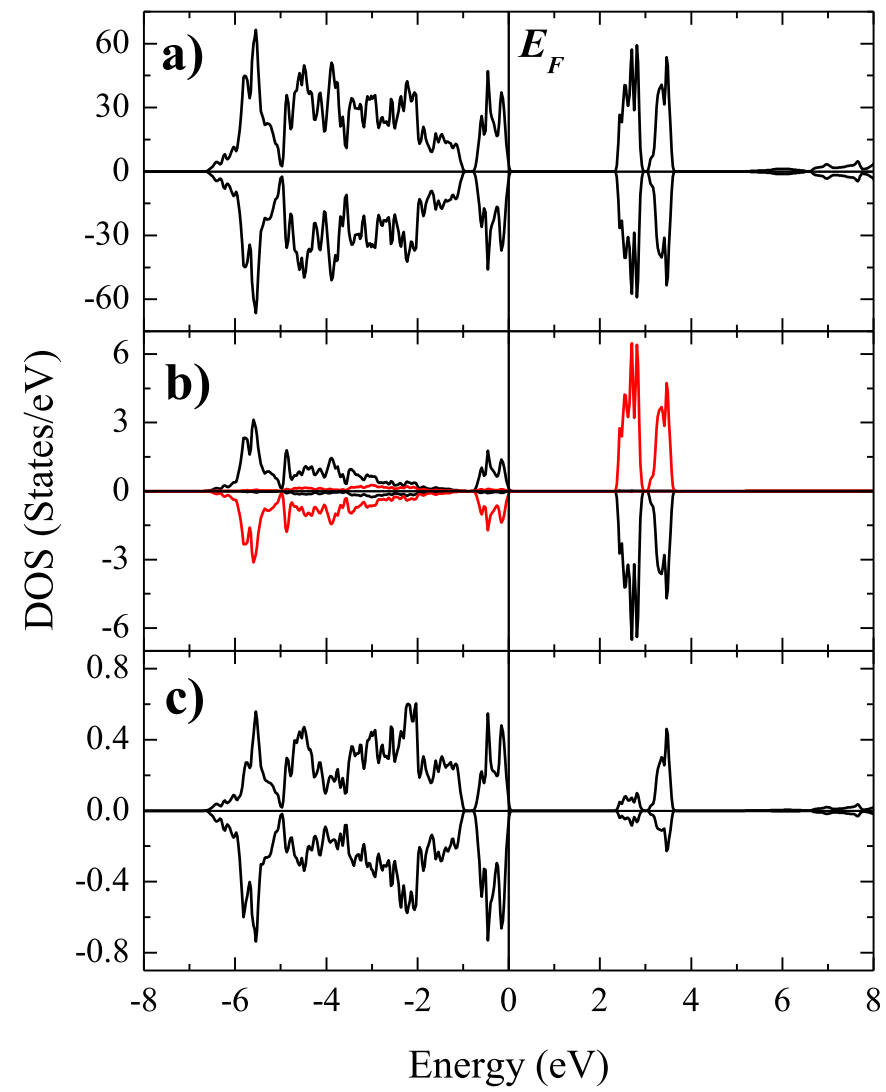

Fig. 6. The total DOS (a) as obtained with the TB-mBJ functional and partial DOS of the dominant angular character of the constituent atoms of ZFO for the AF2 spin arrangement. (b) Fe-3d contribution; (c) 0-2p contributions. Energies are referred to the Fermi level $\left(E_{F}\right)$, denoted as a vertical line. In Figure b), one Fe atom with spin-up and one Fe atom with spin are shown.

As can be seen in Fig. 5, the fully occupied Zn-3d states form a narrow band located in the energy-range $-4.0--6.0 \mathrm{eV}$ (at the bottom of the valence band). In consequence, it is expected that the application of a $U$ value to these states will not produce any important change in the band-structure of the system. In effect, when a $U=5 \mathrm{eV}$ correction is applied to the $\mathrm{Zn}-3 d$ states, the band moves $1 \mathrm{eV}$ to lower energies (compare Fig. $5 \mathrm{c}$ and e) and presents a slightly larger localization. As a consequence, the hybridization with the $0-2 p$ band is reduced. But, as expected, the application of the $U$ correction to the $\mathrm{Zn}-3 d$ states does not produce any change in the band gap (Fig. 5).

\subsection{Hyperfine parameters}

Several previous experimental works have used Mössbauer Spectroscopy (MS) to characterize ZFO (see, for example, Refs. [5,6,13,20,56-60]). Most of them have only detected paramagnetic interactions at room temperature (RT) and two six-line patterns below $10.5 \mathrm{~K}$. In these works, the interpretation of the MS results was based on simple conjectures.

From the theoretical point of view, Mitchell et al. [61] and Schiessl et al. [13] reported calculations of the hyperfine properties at the Fe sites in ZFO. In both cases, the $a b$ initio Hartree-Fock method was employed. The hyperfine properties were calculated using clusters formed by the Fe atoms at the $B$ site and its six nearest-neighbors oxygen. The rest of the system was included in the calculations by considering all the sites beyond the cluster as point charges. This scheme implies an over simplification of the real 
Table 4

Results of ${ }^{57} \mathrm{Fe}$ Mösbauer experiments on $\mathrm{ZnFe}_{2} \mathrm{O}_{4}$ samples at $T_{M}=4.2$ and $300 \mathrm{~K}$ and $a b$ initio predictions for the different magnetic structures considered in this work.

\begin{tabular}{clllll}
\hline & $T_{M}$ & $B_{H F}(\mathrm{~T})$ & $I S(\mathrm{~mm} / \mathrm{s})$ & $\varepsilon(\mathrm{mm} / \mathrm{s})$ & $\Delta Q(\mathrm{~mm} / \mathrm{s})$ \\
\hline $\begin{array}{c}\text { Experimental } \\
\text { results }\end{array}$ & $4.2 \mathrm{~K}[13]$. & $50.57(2)$ & $0.331(2)$ & $0.020(3)$ & - \\
& & $49.66(18)$ & $0.341(5)$ & $0.059(7)$ & \\
Theory, GGA & FM K [20]. & - & $0.350(6)$ & - & $0.333(1)$ \\
& AF2 & 43.0 & 0.62 & 0.04 & 0.24 \\
Theory, GGA+U, & FM & 48.3 & 0.53 & 0.03 & 0.18 \\
$U=5$ eV & AF1 & 51.2 & 0.39 & 0.07 & 0.28 \\
& AF4 & 50.6 & 0.39 & 0.05 & 0.20 \\
& AF3 & 51.3 & 0.38 & 0.05 & 0.22 \\
& AF5 & 50.5 & 0.38 & 0.05 & 0.23 \\
& AF2 & 51.1 & 0.38 & 0.06 & 0.23 \\
\hline
\end{tabular}

system and cannot take into account the complexity of the problem. More recently, we have presented calculations of $\varepsilon$ and $\Delta Q$ at the Fe sites of pristine and normal ZFO in order to discuss the MS results obtained at $300 \mathrm{~K}$ [62]. Only one spin configuration was considered. In the present paper we extend those calculations to different spin arrangements in order to explain some features observed in the experiments performed at $4.2 \mathrm{~K}$. For the sake of simplicity, we will compare the ab initio predictions obtained here with the results at $4.2 \mathrm{~K}$ reported by W. Schiessl et al. [13] and with those reported by Evans at $300 \mathrm{~K}$ [20]. In addition to ${ }^{57} \mathrm{Fe}-\mathrm{MS}, \mathrm{W}$. Schiessl et al. also employed neutron diffraction (ND), muon-spin rotation/relaxation in order to investigate the magnetic properties of ZFO [13]. For that study, samples with Fe located only at site $B$ sites (inversion degree below the limit of detection, see Ref. [13]) were employed. The ND experiments revealed temperatures as large as $85 \mathrm{~K}$ broad diffuse peaks of magnetic origin that were associated to a short-range magnetic order (SRO). Below $10.5 \mathrm{~K}$ antiferromagnetic SRO and antiferromagnetic long-range order (LRO) coexist. The regions exhibiting SRO are very small, in the order of $30 \AA \AA$. The experiments revealed that SRO it is not caused by partial inversion but rather is an intrinsic property of ZFO and the origin of this SRO is still unclear [13].

For pristine and normal ZFO, all the Fe atoms "felt" the same surroundings. In consequence, one might expect that below $10.5 \mathrm{~K}$ the ${ }^{57} \mathrm{Fe}$ Mössbauer spectrum can be fitted with one magnetic sextet. But, in the experiments the better fits to the experimental spectra were obtained when a superposition of two magnetic sextets were considered. One of the sextets $\left(\mathrm{HFI}_{1}\right)$ is characterized by a $B_{H F}$ of $50.57 \mathrm{~T}$. The second interaction $\left(\mathrm{HFI}_{2}\right)$ is characterized by a slightly smaller $B_{H F}$ of $49.66 \mathrm{~T}$ (see Table 4 ). Based in the neutron diffraction and muon-spin rotation/relaxation results the two hyperfine interactions observed below $10.5 \mathrm{~K}$ were attribute to the coexistence of the long and short-range magnetic order $\left(\mathrm{HFI}_{1}\right.$ and $\mathrm{HFI}_{2}$, respectively) [13].

Going to our $a b$ initio predictions, we can see Table 4 that all the antiferromagnetic configurations present similar hyperfine parameters $\left(B_{H F}\right.$ in the order of $51 \mathrm{~T}$, IS of $0.38 \mathrm{~mm} / \mathrm{s}$ and $\varepsilon$ of $0.02-0.03 \mathrm{~mm} / \mathrm{s}$ ). For the case of the ferromagnetic configuration, a smaller $B_{H F}$ is obtained $(48.5 \mathrm{~T})$ and a larger $\varepsilon$ value. These differences obtained for the BHF and $\varepsilon$ values for the FM spin arrangement compared to the AF cases is larger than our precision error. Based on this we can attribute the experimentally observed $\mathrm{HFI}_{1}$ (LRO) to the antiferromagnetic configurations and the $\mathrm{HFI}_{2}$ (SRO) to the ferromagnetic configuration of ZFO. This interpretation of the experimentally observed hyperfine interactions also supports the hypothesis of small clusters of ferromagnetically aligned spins immersed in the long range antiferromagnetic structure, in agreement with the work of Kamazawa et al. [10], who proposed that nearest-neighbor interactions in ZFO are ferromagnetic, while the third-neighbor interactions are antiferromagnetic.
At room temperature $(300 \mathrm{~K})$, the Mössbauer spectra can be fitted with one doublet originating from a quadrupole interaction characterized by $\Delta Q=0.32 \mathrm{~mm} / \mathrm{s}$ and $I S=0.35 \mathrm{~mm} / \mathrm{s}$. Experimental results at $300 \mathrm{~K}$ can only be "crudely" compared with the $a b$ initio predictions (that correspond to $0 \mathrm{~K}$ ). All possible thermal effects are not included in the calculations. Even worse, at $300 \mathrm{~K}$ the compound behaves as a paramagnet and it is not possible to perform an $a b$ initio simulation on a paramagnetic system. We have used the AF2 configuration, which is indeed a weakness of the approach, but taking this comment into account, we can conclude that our calculations correctly reproduce the Mössbauer results at $300 \mathrm{~K}$ (see Table 4).

\section{Conclusions}

In the present work we have studied by means of $a b$ initio GGA and GGA $+U$ calculations the structural, electronic and magnetic properties of ZFO and the hyperfine parameters at the Fe site for different spin arrangements. We show that the plain GGA calculations fail in the prediction of the different properties of ZFO (lattice parameter, band gap, magnetic moments and hyperfine parameters at the Fe sites) and that the GGA $+U$ approach is essential for a good description of the electronic structure of ZFO. To avoid an arbitrary selection of the $U$ parameter, calculations in different Fe-oxides were performed as a function of $U$. From this auxiliary study, we determined that a value of $U=5 \mathrm{eV}$ correctly predicts different properties and hyperfine parameters at the Fe sites of this set of oxides. When the $U=5 \mathrm{eV}$ value is transferred to ZFO, we found a very good agreement theory-experiment for the lattice parameter and the magnetic moment at the Fe sites. Also, a band gap in the order of $2.2 \mathrm{eV}$ is found for ZFO, in agreement with the experiments and with the value reported in other Fe-O systems. Calculations Including a $U$ correction for the $3 d$ levels of $\mathrm{Zn}$ did not affect our results. State-of-the-art TB-mBJ calculations confirm the band structure and the gap obtained in the $\mathrm{GGA}+U$ calculations for $U=5 \mathrm{~V}$.

The lattice parameter of ZFO is predicted to be $8.46 \AA$, in agreement with the experimental results, in the range between 8.43 and $8.46 \AA$. Of all spin-arrangements considered the lowest total energy corresponded to the antiferromagnetic configuration AF2. This configuration consists of a pair of parallel spins aligned antiferromagnetically to another pair at the nearest layer. The energy differences between configurations AF2 and AF3, AF4 and AF5 that also consist of parallel spin clusters are very small. Calculations performed in larger supercells of 112 atoms show that clusters formed by more than two Fe atoms with parallel spins but antiferromagnetically aligned with neighbor clusters have (at least) the same energy that the AF2 configuration. All these facts show that the energy landscape of ZFO is complex and characterized by a number of very closed local energy minima, All these results points to a spin-glass behavior of the ZFO ferrite.

The hyperfine interactions reported by MS experiments at room temperature and a $4.2 \mathrm{~K}$ are also successfully reproduced by our calculations. All the antiferromagnetic configurations present very similar hyperfine parameters (IS, $B_{H F}, \varepsilon$ ) at the Fe sites. On the other hand the ferromagnetic structure presents hyperfine parameters slightly different (smaller $B_{H F}$, larger $\varepsilon$ values). From the comparison with our calculations, the two interactions observed at $T<10.5 \mathrm{~K}$ can be now associated to two different magnetic environments for $\mathrm{Fe}$, in agreement with the model previously discussed: small clusters of spins ferromagnetically coupled arranged antiferromagnetically with other spin clusters.

These results provide a solid base for further studies of the role of defects (inversion, oxygen vacancies) on the electronic and magnetic properties of ZFO. 


\section{Acknowledgments}

This work was partially supported by CONICET (Grants PIP908, PIP01111, and PIP0002), National University of La Plata (Grants X543, X625 and 11/X708), ANPCyT (Grants PICT 00898 and PICT2013-2616), UNNOBA (Project SIB2017 Exp. 0176/2017) and MINCyT-DAAD (Grant DA13/02). Calculations were performed using the Huge Cluster, University of Aarhus, Denmark. The authors want to thank to Dra. M. Weissmann for fruitful discussions and the carefully reading of the manuscript.

\section{References}

[1] J. Smit, H.P.J. Wijn, Ferrites, Philips Technical Library, 1959.

[2] D.C. Johnston, Superconducting and normal state properties of $\mathrm{Li}_{1+x} \mathrm{Ti}_{2-\mathrm{x}} \mathrm{O}_{4}$ spinel compounds. I. Preparation, crystallography, superconducting properties, electrical resistivity, dielectric behavior, and magnetic susceptibility, J. Low. Temp. Phys. 25 (1976) 145-175.

[3] R.W. McCallum, D.C. Johnston, C.A. Luengo, M.B. Maples, Superconducting and normal state properties of $\mathrm{Li}_{1+\mathrm{x}} \mathrm{Ti}_{2-\mathrm{x}} \mathrm{O}_{4}$ spinel compounds. II. Lowtemperature heat capacity, J. Low Temp. Phys. 25 (1976) 177-193.

[4] R.E. Vandenberghe, E. deGrave, in: G.J. Long, F. Grandjean (Eds.), Mössbauer Spectroscopy Applied to Inorganic Chemistry, Plenum, New York, 1989.

[5] M. Niyaifar, Effect of preparation on structure and magnetic properties of $\mathrm{ZnFe}_{2} \mathrm{O}_{4}$, J. Magn. 19 (2014) 101-105.

[6] B. Pandey, F.J. Litterst, E.M. Baggio-Saitovitch, Preferential spin canting in nano size zinc ferrite, J. Magn. Magn. Mater. 385 (2015) 412-417.

[7] Chao Jin, Peng Li, Wenbo Mi, Haili Bai, Structure, magnetic and transport properties of epitaxial $\mathrm{ZnFe}_{2} \mathrm{O}_{4}$ films: an experimental and first principles study, J. App. Phys. 115 (2014) 213908.

[8] Jinhuan Yao, Yanwie Li, Xuanha Li, Xiaodong Zhu, First-Principles study of the geometric and electronic structures of zinc ferrite with vacancy defect, Metall. Mater. Trans. A 47 (2016) 3753-3760 and references there in.

[9] I.A. Vedernikova, Magnetic nanoparticles: advantages of using, methods for preparation, characterization, application in pharmacy, Rev. J. Chem. 5 (2015) $256-280$.

[10] K. Kamazawa, Y. Tsunoda, H. Kadowaki, K. Kohn, Magnetic neutron scattering measurements on a single crystal of frustrated $\mathrm{ZnFe}_{2} \mathrm{O}_{4}$, Phys. Rev. B 68 (2003), 024412 and references there in.

[11] Y. Yamada, K. Kamazawa, Y. Tsunoda, Interspin interactions in $\mathrm{ZnFe}_{2} \mathrm{O}_{4}$ : theoretical analysis of neutron scattering study, Phys. Rev. B 66 (2002), 064401.

[12] K. Kamazawa, Y. Tsunoda, K. Odaka, K. Kohn, Spin liquid state in $\mathrm{ZnFe}_{2} \mathrm{O}_{4}$, J. Phys. Chem. Solids 60 (1999) 1261-1264.

[13] W. Schiessl, W. Potzel, H. Karzel, M. Steiner, G.M. Kalvius, A. Martin, M.K. Krause, I. Halevy, J. Gal, W. Schäfer, G. Will, M. Hillberg, R. Wäppling, Magnetic properties of the $\mathrm{ZnFe}_{2} \mathrm{O}_{4}$ spinel, Phys. Rev. B 53 (1996) 9143-9151.

[14] T. Usa, K. Kamazawa, H. Sekiya, S. Nakamura, Y. Tsunoda, K. Kohn, M. Tanaka, Magnetic properties of $\mathrm{ZnFe}_{2} \mathrm{O}_{4}$ as a 3-D geometrical spin frustration system, J. Phys. Soc. Jpn. 73 (2004) 2834-2840.

[15] K. Tomiyazu, K. Kamazawa, A spin molecule model for geometrically frustrated spinel $\mathrm{ZnFe}_{2} \mathrm{O}_{4}$, J. Phys. Soc. Jpn. 80 (2011). SB024-1 SB024-3.

[16] M.A. Hakim, M. Manjurul Haque, M. Huq, P. Nordblad, Spin-glass-like ordering in the spinel $\mathrm{ZnFe}_{2} \mathrm{O}_{4}$ ferrite, Physica B 406 (2011) 48-51.

[17] V. Cannella, J.A. Midosh, Magnetic ordering in gold-iron alloys, Phys. Rev. B 6 (1972) 4220-4237.

[18] S. Kirkpatrick, D. Sherrington, Infinite-ranged models of spin-glasses, Phys. Rev. B 17 (1978) 4384-4403.

[19] U. Köning, E.F. Bertaut, Y. Gros, G. Chol, Models of the magnetic structure of zinc ferrite, Solid State Commun. 8 (1970) 759-764.

[20] B.J. Evans, S.S. Hafner, H.P. Weberb, Electric field gradients at ${ }^{57} \mathrm{Fe}$ in $\mathrm{ZnFe}_{2} \mathrm{O}_{4}$ and $\mathrm{CdFe}_{2} \mathrm{O}_{4}$, J. Chem. Phys. 55 (1971) 5282-5288.

[21] A. Kremenovića, B. Antić, P. Vulić, J. Blanuša, A. Tomic, $\mathrm{ZnFe}_{2} \mathrm{O}_{4}$ antiferromagnetic structure redetermination, J. Magn. Magn. Mater. 426 (2017) $264-266$.

[22] J.A. Gomes, M.H. Sousa, F.A. Tourinho, J. Mestnik-Filho, R. Itri, J. Depeyrot, Rietveld structure refinement of the cation distribution in ferrite fine particles studied by X-ray powder diffraction, J. Magn. Magn. Mater. 289 (2005) $184-187$.

[23] P. Blaha, K. Schwarz, G. Madsen, D. Kvasnicka, J. Luitz, WIEN2k, an Augmented Plane Wave Plus Local Orbitals Program for Calculating Crystal Properties, Technical Universität Wien, Austria, 1999.

[24] E. Sjöstedt, L. Nordström, D.J. Singh, An alternative way of linearizing the augmented plane-wave method, Solid State Commun. 114 (2000) 15-20.

[25] G.K.H. Madsen, P. Blaha, K. Schwarz, E. Sjöstedt, L. Nordström, Efficient linearization of the augmented plane-wave method, Phys. Rev. B 64 (2001), 195134.

[26] S. Cottenier, Density Functional Theory and the Family of (L)APW-methods: a Step-by-Step Introduction, KU Leuven, Belgium, 2002. http://www.wien2k.at/ reg_user/textbooks.

[27] Z. Wu, R.E. Cohen, More accurate generalized gradient approximation for solids, Phys. Rev. B 73 (2006), 235116.

[28] V.I. Anisimov, I.V. Solovyev, M.A. Korotin, M.T. Czyzyk, G.A. Sawatzky, Densityfunctional theory and $\mathrm{NiO}$ potoemission spectra, Phys. Rev. B 48 (1993) $16929-16934$.

[29] R.C. Albers, N.E. Christensen, A. Svane, Hubbard-U band-structure methods J. Phys. Condens. Matter 21 (2009), 343201.

[30] S. Duhalde, M.F. Vignolo, F. Golmar, C. Chiliotte, C.E. Rodríguez Torres, L.A. Errico, A.F. Cabrera, M. Rentería, F.H. Sanchez, M. Weissmann, Appearance of room-temperature ferromagnetism in Cu-Doped $\mathrm{TiO}_{2-\delta}$ films, Phys. Rev. B 72 (2005), $161313(\mathrm{R})$.

[31] L.S. Errico, K. Lejaeghere, J. Runco, S.N. Mishra, M. Rentería, S. Cottenier, Precision of electric-field gradient predictions by density functional theory and implications for the nuclear quadrupole moment and its error bar of the ${ }^{111} \mathrm{Cd} 245 \mathrm{keV} 5 / 2^{+}$level, J. Phys. Chem. C 120 (2016) 23111-23120.

[32] J.P. Perdew, Y. Wang, Accurate and simple analytic representation of the electron-gas correlation energy, Phys. Rev. B Condens. Matter Mater. Phys. 45 (1992) 13244-13249.

[33] G.N. Darriba, E.L. Muñoz, L.A. Errico, M. Rentería, Ab initio study of structural, electronic, and hyperfine properties of n-type $\mathrm{SnO}_{2}$ :Ta semiconductor, J. Phys Chem. C 118 (2014) 19929-19939.

[34] G. Schatz, A. Weidinger, Nuclear Condensed Matter Physics-Nuclear Methods and Applications, Wiley, Chichester, 1996.

[35] E.N. Kaufmann, R.J. Vianden, The electric field gradient in noncubic metals, Rev. Mod. Phys. 51 (1979) 161-214.

[37] P. Gütlich, E. Bill, A.X. Trautwein, Mössbauer Spectroscopy and Transition Metal Chemistry, Springer-Verlag, Berlin, Heidelberg, 2011.

[38] A.M. Mudarra Navarro, C. Rodríguez Torres, L.A. Errico, Calibration of the isomer shift of the $14.4 \mathrm{keV}$ transition of ${ }^{57} \mathrm{Fe}$, Hyperfine Interact. 202 (2011) $17-121$.

[39] M. El Khalifi, P.E. Lippens, First-Principles investigation of the ${ }^{57} \mathrm{Fe}$ Mössbauer parameters of $\mathrm{LiFePO}_{4}$ and $\mathrm{FePO}_{4}$, J. Phys. Chem. C 120 (2016) 28375-28389.

[40] P.H. Borcherds, C.V. Sheth, Least squares fitting of a straight line to a set of data points, Eur. J. Phys. 16 (1995) 204-210.

[41] C.V. Sheth, A. Ngwengwe, P.H. Borcherds, Least squares fitting of a straight line to a set of data points: II. Parameter variances, Eur. J. Phys. 17 (1996) $322-326$.

[42] G. Martínez-Pinedo, P. Schwerdtfeger, E. Caurier, K. Langanke, W. Nazarewicz T. Söhnel, Nuclear quadrupole moment of ${ }^{57} \mathrm{Fe}$ from microscopic nuclear and atomic calculations, Phys. Rev. Lett. 87 (2001), 062701.

[43] R. Coehoorn, Improved analysis of hyperfine fields in $\mathrm{Fe}$, Co and $\mathrm{Ni}$, and application to orbital magnetism in intermetallic compounds, J. Magn. Magn. Mater. 159 (1996) 55-63.

[44] P. Novák, J. Kuneš, W.E. Pickett, W. Ku, F.R. Wagner, Self-interaction correction and contact hyperfine field, Phys. Rev. B 67 (2003), 140403.

[45] H. Akai, T. Kotani, Theory of hyperfine fields of iron, Hyperfine Interact. (1999) 120.

[46] U. Lundin, O. Eriksson, Novel method of self-interaction corrections in density functional calculations, Int. J. Quant. Chem. 81 (2001) 247-252.

[47] P. Novak, V. Chlan, Contact hyperfine field at Fe nuclei from density functional calculations, Phys. Rev. B 81 (2010), 174412.

[48] M. Darby Dyar, D.G. Agresti, M.W. Schaefer, Mössbauer spectroscopy of earth and planetary materials, Annu. Rev. Earth Planet. Sci. 125 (2006) 34-83. Christopher A. Grant,5 and Elizabeth C. Sklute2.

[49] J. Singh, M. Gupta, R. Gupta, Density-functional description of spinel $\mathrm{ZnFe}_{2} \mathrm{O}_{4}$, Phys. Rev. B 63 (2001), 205102.

[50] S. Soliman, A. Elfalaky, H. Fecher, C. Felser, Electronic structure calculations for $\mathrm{ZnFe}_{2} \mathrm{O}_{4}$, Phys. Rev. B 83 (2011), 085205.

[51] A. Manikandan, J. Judith Vijaya, M. Sundararajan, C. Meganathan, L. John Kennedy, M. Bououdina, Optical and magnetic properties of Mg-doped $\mathrm{ZnFe}_{2} \mathrm{O}_{4}$ nanoparticles prepared by rapid microwave combustion method, Superlattices Microstruct. 64 (2013) 118-131.

[52] J. Prakash, Effect of aluminium substitution on electrical conductivity and physical properties of zinc ferrite, Mater. Sci. Lett. 6 (1987) 651-652.

[53] M. El-Shabasy, DC electrical properties of Zn-Ni ferrites, J. Magn. Magn. Mater. 172 (1997) 188-192.

[54] A.D. Becke, E.R. Johnson, A simple effective potential for exchange, J. Chem. Phys. 124 (2006) 221101.

[55] F. Tran, P. Blaha, Accurate band gaps of semiconductors and insulators with a semilocal exchange-correlation potential, Phys. Rev. Lett. 102 (2009) 226401.

[56] G.F. Goya, E.R. Leite, Ferrimagnetism and spin canting of $\mathrm{Zn}^{57} \mathrm{Fe}_{2} \mathrm{O}_{4}$ nanoparticles embedded in ZnO matrix, J. Phys. Condens. Matter 15 (2003) 641-651.

[57] M.J. Nasr Isfahani, V. Sepelak, A Mössbauer effect study, as the main key to the investigation of anomalous magnetic properties of MnZn nanoferrite, Iran. J. Phys. Res. 12 (2012) 33-37.

[58] S.J. Stewart, S.J.A. Figueroa, M.B. Sturla, R.B. Scorzelli, F. García, F.G. Requejo Magnetic $\mathrm{ZnFe}_{2} \mathrm{O}_{4}$ nanoferrites studied by X-ray magnetic circular dichroism and Mössbauer spectroscopy, Physica B 389 (2007) 155-158.

[59] J. Battle, T. Clark, B. Evans, ${ }^{57}$ Fe Mössbauer spectroscopy of zinc ferrite prepared by a variety of synthetic methods, J. de Phys. IV Colloque 07 (C1) (1997) C1-257-C1-258. 
[60] R.C. Srivastava, J.P. Singh, H.M. Agrawal, R. Kumar, A. Tripathi, R.P. Tripathi, V.R. Reddy, A. Gupta, ${ }^{57} \mathrm{Fe}$ Mössbauer investigation of nanostructured zinc ferrite irradiated by $100 \mathrm{MeV}$ oxygen beam, J. Phys. Conf. Ser. 217 (2010), 012109.

[61] D.W. Mitchell, T.P. Das, W. Potzel, W. Schiessl, H. Karzel, M. Steiner, M. Köfferlein, U. Hiller, G.M. Kalvius, A. Martin, W. Schäfer, G. Will, I. Halevy,
J. Gal, Ab initio electric-field gradients and electron densities at ${ }^{27} \mathrm{Al},{ }^{57} \mathrm{Fe}$, and ${ }^{67} \mathrm{Zn}$ in the spinels $\mathrm{ZnAl}_{2} \mathrm{O}_{4}$ and $\mathrm{ZnFe}_{2} \mathrm{O}_{4}$, Phys. Rev. B 53 (1996) 7684-7698.

[62] J.M. Quintero, K.L. Salcedo Rodríguez, G.A. Pasquevich, P. Mendoza Zélis, S.J. Stewart, C. Rodríguez Torres, L.A. Errico, Experimental and $a b$ initio study of the hyperfine parameters of $\mathrm{ZnFe}_{2} \mathrm{O}_{4}$ with defects, Hyperfine Interact. 237 (2016) 63. 